SINP-TNP/97-02

hep-th/9705016

\title{
On S-Duality of Toroidally Compactified Type IIB String Effective Action
}

\author{
Shibaji Roy \\ Saha Institute of Nuclear Physics \\ 1/AF Bidhannagar, Calcutta 700 064, India
}

\begin{abstract}
It has been shown recently that the toroidally compactified type IIB string effective action possesses an $\mathrm{SL}(2, \mathrm{R})$ invariance as a consequence of the corresponding symmetry in ten dimensions when the self-dual five-form field strength is set to zero. By working in the string frame we clarify how a $Z_{2}$ subgroup of this $\mathrm{SL}(2, \mathrm{R})$ group responsible for producing the strong-weak coupling duality in the ten dimensional theory produces the same symmetry for the reduced theory. In the absence of the full covariant action of type IIB supergravity theory, we show that the T-dual version of type IIA string effective action (including the $\mathrm{R}-\mathrm{R}$ terms) in $\mathrm{D}=9$ also possesses the $\mathrm{SL}(2, \mathrm{R})$ invariance indicating that this symmetry is present for the full type IIB string effective action compactified on torus.
\end{abstract}

\footnotetext{
*E-mail address: roy@tnp.saha.ernet.in
} 
By now there is a mounting evidence in support of the conjecture that type IIB superstring theory in ten dimensions has an $\mathrm{SL}(2, \mathrm{R})$ invariance [1-3]. The discrete subgroup of this $\mathrm{SL}(2, \mathrm{R})$ group survives as an exact symmetry of the quantum theory and has been referred to as S-duality in the literature in analogy with the corresponding symmetry in $\mathrm{N}=4, \mathrm{D}=4$ heterotic string theory [4,5]. Under this $\mathrm{SL}(2, \mathrm{R})$ transformation the ten dimensional dilaton of type IIB string theory transforms non-trivially and so it mixes up the different orders of the string perturbation theory. In particular, a $\mathrm{Z}_{2}$ subgroup of this $\mathrm{SL}(2, \mathrm{R})$ group relates the weak and the strong coupling regime of the type IIB string theory in $\mathrm{D}=10$, when the scalar field in the Ramond-Ramond ( $\mathrm{R}$ $\mathrm{R}$ ) sector of the spectrum is set to zero. Thus this symmetry is non-perturbative and that is why it is difficult to prove this conjecture. Many interesting consequences of this symmetry have been explored in refs.[3,6,7]. In particular, the various classical p-brane solutions of type IIB string theory have been shown to form $\operatorname{SL}(2, \mathrm{Z})$ multiplets and as a consequence the existence of bound states of $n$ fundamental p-branes with $m$ Dirichlet p-branes with $(n, m)$ relatively prime integers, has been predicted in ref.[8]. Although it is difficult to prove this conjecture, there are various indirect ways to understand the origin of this symmetry. Purely, from string theory point of view the SL(2, R) symmetry can be understood from the ten-dimensional string-string duality conjecture of Type I and heterotic string theory with gauge group $\mathrm{SO}(32)$ and T-duality [2,9]. This symmetry can also be understood from the hypothetical higher dimensional theories called 'M-theory' $[6,10]$ and 'F-theory' [11] compactified on torus.

In this paper, we will study the toroidal compactification $[12,13]$ of type IIB string theory and explore some of the consequences of $\mathrm{SL}(2, \mathrm{R})$ invariance of ten dimensional theory. Since the ten dimensional theory already has $\operatorname{SL}(2, \mathrm{R})$ invariance, it is expected that this symmetry will persist also in lower dimensions. We will first show that it is indeed the case. It is well-known $[14,15]$ that the full type IIB supergravity theory is nonLagrangian because of the presence of a self-dual four-form gauge field in the spectrum. But, once we set the corresponding field strength to zero, a consistent covariant action can be written from which the field equations of type IIB supergravity theory could be obtained [2]. We will take this type IIB string effective action and reduce it on (10 - D) dimensional torus in the string frame. This D-dimensional effective action when written down in the Einstein frame will be shown to possess an $\operatorname{SL}(2, \mathrm{R})$ invariance as expected. The toroidal compactification of the same type IIB string effective action in the 
Einstein frame has been studied recently by Maharana [16] and the $\operatorname{SL}(2, \mathrm{R})$ invariance of the reduced action was shown to follow directly in this way. We will point out that in showing this invariance of the toroidally compactified theory, it is not the D-dimensional dilaton but rather a linear combination of the dilaton and other moduli of the theory which appears in the matrix $\mathcal{M}_{\mathrm{D}}$ (see eq.(19) and the discussion after eq.(21)). It is for this reason, a $\mathrm{Z}_{2}$ subgroup of the $\mathrm{SL}(2, \mathrm{R})$ group which is responsible for producing the strong-weak coupling duality symmetry in the ten dimensional theory does not necessarily imply the same symmetry in the reduced theory. We will clarify how the strong-weak coupling duality symmetry can be understood in the reduced theory by working in the string frame. It should be emphasized that this effect becomes transparent if we reduce the action in the string frame and then go over to the Einstein frame rather than reducing the theory directly in the Einstein frame as in ref.[16]. Then we will show that not only this truncated version of the type IIB string effective action but also the full action has the $\mathrm{SL}(2, \mathrm{R})$ invariance when compactified on torus. In the absence of the full action, we will take the following strategy to understand this symmetry in the full theory. Since the complete type IIA string effective action including the R-R terms is known $[17,18]$, we reduce this action on a circle. It should be pointed out here that it is difficult to study the toroidal compactification of this action in general because of the presence of the topological terms whose forms are very specific to the dimensionality of the reduced theory. We will therefore look at the simplest case i.e. the reduction on a circle. Since in $\mathrm{D}=9$, type IIA and type IIB theories are T-dual to each other [19,20], we will take the $\mathrm{T}$-dual version of this $\mathrm{D}=9$ type IIA action and then show that this action is indeed $\mathrm{SL}(2, \mathrm{R})$ invariant. Thus we conclude that the full type IIB string effective action when toroidally compactified also possesses an $\mathrm{SL}(2, \mathrm{R})$ invariance.

Let us recall that the massless spectrum of the type IIB string theory in the bosonic sector contains a graviton $\hat{g}_{B, \hat{\mu} \hat{\nu}}$, a dilaton $\hat{\phi}^{(1)}$ and an antisymmetric tensor field $\hat{b}_{\hat{\mu} \hat{\nu}}^{(1)}$ as Neveu-Schwarz-Neveu-Schwarz (NS-NS) sector states whereas in the R-R sector it contains another scalar $\hat{\phi}^{(2)}$, another antisymmetric tensor field $\hat{b}_{\hat{\mu} \hat{\nu}}^{(2)}$ and a four-form gauge field $\hat{A}_{\hat{\mu} \hat{\nu} \hat{\rho} \hat{\sigma}}^{+}$whose field strength is self-dual [21] the four-form gauge field is set to zero, the type IIB supergravity equation of motion can

\footnotetext{
*We denote the ten dimensional space-time coordinates and the fields with a 'hat'. The objects in lower dimensions will be denoted without 'hat'.
} 
be obtained from the following covariant action [2]:

$$
\begin{aligned}
S_{\mathrm{IIB}}^{(10)}=\int d^{10} \hat{x} \sqrt{-\hat{g}_{B}}\left[e^{-2 \hat{\phi}^{(1)}}\left(\hat{R}_{B}+4 \partial_{\hat{\mu}} \hat{\phi}^{(1)} \partial^{\hat{\mu}} \hat{\phi}^{(1)}-\frac{1}{12} \hat{h}_{\hat{\mu} \hat{\nu} \hat{\lambda}}^{(1)} \hat{h}^{(1) \hat{\mu} \hat{\nu} \hat{\lambda}}\right)\right. \\
\left.-\frac{1}{2} \partial_{\hat{\mu}} \hat{\phi}^{(2)} \partial^{\hat{\mu}} \hat{\phi}^{(2)}-\frac{1}{12}\left(\hat{h}_{\hat{\mu} \hat{\nu} \hat{\lambda}}^{(2)}+\hat{\phi}^{(2)} \hat{h}_{\hat{\mu} \hat{\nu} \hat{\lambda}}^{(1)}\right)\left(\hat{h}^{(2) \hat{\mu} \hat{\nu} \hat{\lambda}}+\hat{\phi}^{(2)} \hat{h}^{(1) \hat{\mu} \hat{\nu} \hat{\lambda}}\right)\right]
\end{aligned}
$$

where

$$
\hat{h}_{\hat{\mu} \hat{\nu} \hat{\lambda}}^{(i)}=\left(\partial_{\hat{\mu}} \hat{b}_{\hat{\nu} \hat{\lambda}}^{(i)}+\text { cyc. in } \hat{\mu} \hat{\nu} \hat{\lambda}\right), \quad i=1,2
$$

The action in (1) is known to possess a global $\mathrm{SL}(2, \mathrm{R})$ invariance which can be better understood in the Einstein frame where the Einstein metric and the string metric are related as $\overline{\hat{g}}_{B, \hat{\mu} \hat{\nu}}=e^{-\frac{1}{2} \hat{\phi}^{(1)}} \hat{g}_{B, \hat{\mu} \hat{\nu}}$. In the Einstein frame the action can be written as:

$$
\begin{aligned}
\bar{S}_{\mathrm{IIB}}^{(10)=} & \int d^{10} \hat{x} \sqrt{-\overline{\hat{g}}_{B}}\left[\overline{\hat{R}}_{B}-\frac{1}{2} \partial_{\hat{\mu}} \hat{\phi}^{(1)} \partial^{\hat{\mu}} \hat{\phi}^{(1)}-\frac{1}{2} e^{2 \hat{\phi}^{(1)}} \partial_{\hat{\mu}} \hat{\phi}^{(2)} \partial^{\hat{\mu}} \hat{\phi}^{(2)}\right. \\
& \left.-\frac{1}{12}\left(e^{-\hat{\phi}^{(1)}} \hat{h}_{\hat{\mu} \hat{\nu} \hat{\lambda}}^{(1)} \hat{h}^{(1) \hat{\mu} \hat{\nu} \hat{\lambda}}+e^{\hat{\phi}^{(1)}}\left(\hat{h}_{\hat{\mu} \hat{\nu} \hat{\lambda}}^{(2)}+\hat{\phi}^{(2)} \hat{h}_{\hat{\mu} \hat{\nu} \hat{\lambda}}^{(1)}\right)\left(\hat{h}^{(2) \hat{\mu} \hat{\nu} \hat{\lambda}}+\hat{\phi}^{(2)} \hat{h}^{(1) \hat{\mu} \hat{\nu} \hat{\lambda}}\right)\right)\right]
\end{aligned}
$$

This action can now be expressed in a manifestly $\mathrm{SL}(2, \mathrm{R})$ invariant form as given below:

$$
\bar{S}_{\mathrm{IIB}}^{(10)}=\int d^{10} \hat{x} \sqrt{-\overline{\hat{g}}_{B}}\left[\overline{\hat{R}}_{B}+\frac{1}{4} \operatorname{tr} \partial_{\hat{\mu}} \hat{\mathcal{M}} \partial^{\hat{\mu}} \hat{\mathcal{M}}^{-1}-\frac{1}{12} \hat{\mathbf{h}}_{\hat{\mu} \hat{\nu} \hat{\lambda}}^{T} \hat{\mathcal{M}} \hat{\mathbf{h}}^{\hat{\mu} \hat{\nu} \hat{\lambda}}\right]
$$

where,

$$
\hat{\mathcal{M}} \equiv\left(\begin{array}{cc}
\left(\hat{\phi}^{(2)}\right)^{2} e^{\hat{\phi}^{(1)}}+e^{-\hat{\phi}^{(1)}} & \hat{\phi}^{(2)} e^{\hat{\phi}^{(1)}} \\
\hat{\phi}^{(2)} e^{\hat{\phi}^{(1)}} & e^{\hat{\phi}^{(1)}}
\end{array}\right)
$$

represents an $\operatorname{SL}(2, \mathrm{R})$ matrix and $\hat{\mathbf{h}}_{\hat{\mu} \hat{\nu} \hat{\lambda}} \equiv\left(\begin{array}{c}\hat{h}_{\hat{\mu} \hat{\nu} \hat{\lambda}}^{(1)} \\ \hat{h}_{\hat{\mu} \hat{\nu} \hat{\lambda}}^{(2)}\end{array}\right)$. Also, the superscript ' $T$ ' represents the transpose of a matrix. The action (4) can be easily seen to be invariant under a global $\mathrm{SL}(2, \mathrm{R})$ transformation $\hat{\mathcal{M}} \rightarrow \Lambda \hat{\mathcal{M}} \Lambda^{T}$ and $\left(\begin{array}{c}\hat{b}_{\hat{\mu} \hat{\nu}}^{(1)} \\ \hat{b}_{\hat{\mu} \hat{\nu}}^{(2)}\end{array}\right) \equiv \hat{\mathbf{b}}_{\hat{\mu} \hat{\nu}} \rightarrow\left(\Lambda^{-1}\right)^{T} \hat{\mathbf{b}}_{\hat{\mu} \hat{\nu}}$, where $\Lambda=$ $\left(\begin{array}{ll}a & b \\ c & d\end{array}\right)$, with $a d-b c=1$, represents a global $\mathrm{SL}(2, \mathrm{R})$ transformation matrix. Note that the canonical metric $\overline{\hat{g}}_{B \hat{\mu} \hat{\nu}}$ remains invariant under the $\mathrm{SL}(2, \mathrm{R})$ transformation. With these transformations the complex scalar field $\hat{\rho}=\left(\hat{\phi}^{(2)}+i e^{-\hat{\phi}^{(1)}}\right)$ undergoes a fractional linear transformation whereas the two two-form potentials $\hat{b}_{\hat{\mu} \hat{\nu}}^{(1)}$ and $\hat{b}_{\hat{\mu} \hat{\nu}}^{(2)}$ transform linearly [3]. In particular, choosing $\Lambda=\left(\begin{array}{cc}0 & 1 \\ -1 & 0\end{array}\right)$ and $\hat{\phi}^{(2)}=0$, the string coupling constant $e^{\hat{\phi}^{(1)}}$ transforms to its inverse showing a strong-weak coupling duality symmetry in the theory.

We now perform the dimensional reduction of the action (1) on $(10-\mathrm{D})$ dimensional torus. In order to achieve this we first split the 10-dimensional coordinates $\hat{x}^{\hat{\mu}}$ to $\mathrm{D}$ 
space-time coordinates $x^{\mu}$ and $(10-\mathrm{D})$ internal coordinates $x^{m}$ and demand that all the D-dimensional fields be independent of the compact coordinates $[12,13]$. By using the Lorentz invariance the ten dimensional vielbein is usually taken in the following triangular form $[22]$

$$
\hat{e}_{\hat{\mu}}^{\hat{\alpha}}=\left(\begin{array}{cc}
e_{\mu}^{\alpha} & a_{\mu}^{(3) n} e_{n}^{a} \\
0 & e_{m}^{a}
\end{array}\right)
$$

where $e_{\mu}^{\alpha}$ and $e_{m}^{a}$ are respectively the space-time and internal vielbeins. $a_{\mu}^{(3) n}$ are (10 - D) vector gauge fields resulting from the dimensional reduction of the metric. (We have reserved $a_{\mu}^{(1) n}$ and $a_{\mu}^{(2) n}$ to denote the gauge fields resulting from the dimensional reduction of the two antisymmetric tensor fields $\hat{b}_{\hat{\mu} \hat{\nu}}^{(1)}$ and $\hat{b}_{\hat{\mu} \hat{\nu}}^{(2)}$.) The D-dimensional metric and the internal metric are given respectively by $g_{B, \mu \nu}=e_{\mu}^{\alpha} e_{\nu}^{\beta} \eta_{\alpha \beta}$ and $g_{m n}=e_{m}^{a} e_{n}^{b} \delta_{a b}$, where our convention for the signature of the Lorentz metric is $(-,+,+, \cdots)$. Note that with this convention $(6), \sqrt{-\hat{g}_{B}}=\sqrt{-g_{D}} \Delta$, where $\hat{g}_{B}=\left(\operatorname{det} \hat{g}_{B, \hat{\mu} \hat{\nu}}\right), g_{D}=\left(\operatorname{det} g_{D, \mu \nu}\right)$ and $\Delta^{2}=\left(\operatorname{det} g_{m n}\right)$. With these definitions the scalar part of the action (1) reduces to [18]:

$$
\begin{aligned}
& \int d^{10} \hat{x} \sqrt{-\hat{g}_{B}} {\left[e^{-2 \hat{\phi}^{(1)}}\left(\hat{R}_{B}+4 \partial_{\hat{\mu}} \hat{\phi}^{(1)} \partial^{\hat{\mu}} \hat{\phi}^{(1)}\right)-\frac{1}{2} \partial_{\hat{\mu}} \hat{\phi}^{(2)} \partial^{\hat{\mu}} \hat{\phi}^{(2)}\right] } \\
& \longrightarrow \int d^{D} x \sqrt{-g_{D}}\left[e ^ { - 2 \phi _ { D } ^ { ( 1 ) } } \left(R_{D}+4 \partial_{\mu} \phi_{D}^{(1)} \partial^{\mu} \phi_{D}^{(1)}-\frac{1}{4} g_{m n} f_{\mu \nu}^{(3) m} f^{(3) \mu \nu, n}\right.\right. \\
&\left.\left.+\frac{1}{4} \partial_{\mu} g_{m n} \partial^{\mu} g^{m n}\right)-\frac{1}{2} \Delta \partial_{\mu} \phi^{(2)} \partial^{\mu} \phi^{(2)}\right]
\end{aligned}
$$

where $R_{D}$ is the scalar curvature for the D-dimensional metric $g_{D, \mu \nu}, \hat{\phi}^{(1)}=\phi_{D}^{(1)}+\frac{1}{2} \log \Delta$, $\phi_{D}^{(1)}$ being the D-dimensional dilaton and $\hat{\phi}^{(2)}=\phi^{(2)}$. Also, $f_{\mu \nu}^{(3) m}=\partial_{\mu} a_{\nu}^{(3) m}-\partial_{\nu} a_{\mu}^{(3) m}$. The reduction of the terms involving square of the field strengths associated with the antisymmetric tensor fields $\hat{b}_{\hat{\mu} \hat{\nu}}^{(i)}$ in the action (1) is given below:

$$
\begin{gathered}
-\frac{1}{12} \int d^{10} \hat{x} \sqrt{-\hat{g}_{B}}\left[e^{-2 \hat{\phi}^{(1)}} \hat{h}_{\hat{\mu} \hat{\nu} \hat{\lambda}}^{(1)} \hat{h}^{(1) \hat{\mu} \hat{\nu} \hat{\lambda}}+\left(\hat{h}_{\hat{\mu} \hat{\nu} \hat{\lambda}}^{(2)}+\hat{\phi}^{(2)} \hat{h}_{\hat{\mu} \hat{\nu} \hat{\lambda}}^{(1)}\right)\left(\hat{h}^{(2) \hat{\mu} \hat{\nu} \hat{\lambda}}+\hat{\phi}^{(2)} \hat{h}^{(1) \hat{\mu} \hat{\nu} \hat{\lambda}}\right)\right] \\
\longrightarrow \int d^{D} x \sqrt{-g_{D}}\left[e^{-2 \phi_{D}}\left(-\frac{1}{4} g^{m p} g^{n q} \partial_{\mu} b_{m n}^{(1)} \partial^{\mu} b_{p q}^{(1)}-\frac{1}{4} g^{m p} h_{\mu \nu m}^{(1)} h_{p}^{(1) \mu \nu}-\frac{1}{12} h_{\mu \nu \lambda}^{(1)} h^{(1) \mu \nu \lambda}\right)\right. \\
-\frac{1}{4} \Delta g^{m p} g^{n q}\left(\partial_{\mu} b_{m n}^{(2)}+\phi^{(2)} \partial_{\mu} b_{m n}^{(1)}\right)\left(\partial^{\mu} b_{p q}^{(2)}+\phi^{(2)} \partial^{\mu} b_{p q}^{(1)}\right) \\
-\frac{1}{4} \Delta g^{m p}\left(h_{\mu \nu m}^{(2)}+\phi^{(2)} h_{\mu \nu m}^{(1)}\right)\left(h_{p}^{(2) \mu \nu}+\phi_{p}^{(2)} h_{p}^{(1) \mu \nu}\right) \\
\left.-\frac{1}{12} \Delta\left(h_{\mu \nu \lambda}^{(2)}+\phi^{(2)} h_{\mu \nu \lambda}^{(1)}\right)\left(h^{(2) \mu \nu \lambda}+\phi^{(2)} h^{(1) \mu \nu \lambda}\right)\right]
\end{gathered}
$$

\footnotetext{
${ }^{\dagger}$ Here the Greek letters $(\lambda, \mu, \ldots)$ in the later part of the alphabet denote the curved space-time indices whereas $(\alpha, \beta, \ldots)$ in the beginning of the alphabet correspond to flat tangent space indices. Similarly, the Latin letters $(m, n, \ldots)$ represent the internal indices and $(a, b, \ldots)$ denote the corresponding tangent space indices.
} 
Our definitions and the reduced form of various gauge fields are:

$$
\begin{aligned}
& \hat{g}_{B, \hat{\mu} \hat{\nu}} \longrightarrow\left\{\begin{array}{l}
\hat{g}_{B, m n}=g_{m n} \\
g_{B, \mu m}=\hat{g}_{B, \mu m}=a_{\mu}^{(3) n} g_{m n} \\
\hat{g}_{B, \mu \nu}=g_{B, \mu \nu}+g_{m n} a_{\mu}^{(3) m} a_{\nu}^{(3) n}
\end{array}\right. \\
& \hat{\phi}^{(1)}=\phi_{D}^{(1)}+\frac{1}{2} \log \Delta \\
& \hat{\phi}^{(2)}=\phi^{(2)} \\
& \hat{b}_{\hat{\mu} \hat{\nu}}^{(i)} \longrightarrow\left\{\begin{array}{l}
b_{\mu m}^{(i)}=a_{\mu m}^{(i)}=\hat{b}_{\mu m}^{(i)}+b_{m n}^{(i)} a_{\mu}^{(3) n} \\
b_{\mu \nu}^{(i)}=\hat{b}_{\mu \nu}^{(i)}+a_{\mu}^{(3) m} a_{\nu m}^{(i)}-a_{\nu}^{(3) m} a_{\mu m}^{(i)}-a_{\mu}^{(3) m} b_{m n}^{(i)} a_{\nu}^{(3) n}
\end{array}\right.
\end{aligned}
$$

The corresponding field strengths are given as,

$$
\begin{aligned}
& h_{\mu m n}^{(i)}=\hat{h}_{\mu m n}^{(i)}=\partial_{\mu} b_{m n}^{(i)} \\
& h_{\mu \nu m}^{(i)}=f_{\mu \nu m}^{(i)}-b_{m n}^{(i)} f_{\mu \nu}^{(3) n}
\end{aligned}
$$

where

$$
f_{\mu \nu m}^{(i)}=\partial_{\mu} a_{\nu m}^{(i)}-\partial_{\nu} a_{\mu m}^{(i)}
$$

and finally,

$$
h_{\mu \nu \lambda}^{(i)}=\partial_{\mu} b_{\nu \lambda}^{(i)}-f_{\mu \nu}^{(3) m} a_{\lambda m}^{(i)}+\text { cyc. in } \mu \nu \lambda
$$

It is well-known that the NS-NS sector of the action (1) possesses a global non-compact symmetry $\mathrm{O}(10-\mathrm{D}, 10-\mathrm{D})$ when compactified on $(10-\mathrm{D})$ dimensional torus [23]. In order to show this invariance it was pointed out in ref.[18], that it is necessary to express $h_{\mu \nu \lambda}^{(1)}$ in (16) in terms of $\bar{b}_{\mu \nu}^{(1)} \equiv b_{\mu \nu}^{(1)}-\frac{1}{2}\left(a_{\mu}^{(3)} m a_{\nu m}^{(1)}-a_{\nu}^{(3) m} a_{\mu m}^{(1)}\right)$ as this remains invariant under $\mathrm{O}(10-\mathrm{D}, 10-\mathrm{D})$ transformation. In the present context, the introduction of 'barred' fields is not necessary. So, the complete form of the dimensionally reduced action (1) to D-dimensions is given as:

$$
\begin{gathered}
\int d^{D} x \sqrt{-g_{D}}\left[e ^ { - 2 \phi _ { D } ^ { ( 1 ) } } \left(R_{D}+4 \partial_{\mu} \phi_{D}^{(1)} \partial^{\mu} \phi_{D}^{(1)}-\frac{1}{4} g_{m n} f_{\mu \nu}^{(3) m} f^{(3) \mu \nu, n}+\frac{1}{4} \partial_{\mu} g_{m n} \partial^{\mu} g_{m n}\right.\right. \\
\left.-\frac{1}{4} g^{m p} g^{n q} \partial_{\mu} b_{m n}^{(1)} \partial^{\mu} b_{p q}^{(1)}-\frac{1}{4} g^{m p} h_{\mu \nu m}^{(1)} h_{p}^{(1) \mu \nu}-\frac{1}{12} h_{\mu \nu \lambda}^{(1)} h^{(1) \mu \nu \lambda}\right) \\
-\frac{1}{2} \Delta \partial_{\mu} \phi^{(2)} \partial^{\mu} \phi^{(2)}-\frac{1}{4} \Delta g^{m p} g^{n q}\left(\partial_{\mu} b_{m n}^{(2)}+\phi^{(2)} \partial_{\mu} b_{m n}^{(1)}\right)\left(\partial^{\mu} b_{p q}^{(2)}+\phi^{(2)} \partial^{\mu} b_{p q}^{(1)}\right) \\
-\frac{1}{4} \Delta g^{m p}\left(h_{\mu \nu m}^{(2)}+\phi^{(2)} h_{\mu \nu m}^{(1)}\right)\left(h_{p}^{(2) \mu \nu}+\phi^{(2)} h_{p}^{(1) \mu \nu}\right) \\
\left.-\frac{1}{12} \Delta\left(h_{\mu \nu \lambda}^{(2)}+\phi^{(2)} h_{\mu \nu \lambda}^{(1)}\right)\left(h^{(2) \mu \nu \lambda}+\phi^{(2)} h^{(1) \mu \nu \lambda}\right)\right]
\end{gathered}
$$

We have obtained the reduced action in the string frame metric and the D-dimensional string coupling constant is given by $\lambda_{D} \sim e^{\phi_{D}^{(1)}}$, where $\phi_{D}^{(1)}$ is the D-dimensional dilaton 
whose relation with the ten dimensional dilaton is given in eq.(10). Just like the ten dimensional case, the $\mathrm{SL}(2, \mathrm{R})$ invariance of the action (17) becomes manifest in the Einstein frame. In order to rewrite the action in the Einstein frame we rescale the string metric as usual by $\bar{g}_{D, \mu \nu}=e^{-\frac{4}{D-2} \phi_{D}^{(1)}} g_{D, \mu \nu}$. Then the action (17) takes the following form:

$$
\begin{array}{rl}
\int d^{D} & x \sqrt{-\bar{g}_{D}}\left[\bar{R}_{D}-\frac{1}{2} \partial_{\mu} \bar{\phi}_{D}^{(1)} \partial^{\mu} \bar{\phi}_{D}^{(1)}-\frac{1}{2} e^{2 \bar{\phi}_{D}^{(1)}} \partial_{\mu} \phi^{(2)} \partial^{\mu} \phi^{(2)}+\frac{1}{8} \partial_{\mu} \log \bar{\Delta} \partial^{\mu} \log \bar{\Delta}\right. \\
& +\frac{1}{4} \partial_{\mu} \bar{g}_{m n} \partial^{\mu} \bar{g}^{m n}-\frac{1}{4} \bar{g}_{m n} f_{\mu \nu}^{(3) m} f^{(3) \mu \nu, n}-\frac{1}{4}(\bar{\Delta})^{1 / 2} \bar{g}^{m p} \bar{g}^{n q} e^{-\bar{\phi}_{D}^{(1)}} \partial_{\mu} b_{m n}^{(1)} \partial^{\mu} b_{p q}^{(1)} \\
& -\frac{1}{4}(\bar{\Delta})^{1 / 2} \bar{g}^{m p} \bar{g}^{n q} e^{\bar{\phi}_{D}^{(1)}}\left(\partial_{\mu} b_{m n}^{(2)}+\phi^{(2)} \partial_{\mu} b_{m n}^{(1)}\right)\left(\partial^{\mu} b_{p q}^{(2)}+\phi^{(2)} \partial^{\mu} b_{p q}^{(1)}\right) \\
& -\frac{1}{4}(\bar{\Delta})^{1 / 2} \bar{g}^{m p}\left\{e^{-\bar{\phi}_{D}^{(1)}} h_{\mu \nu m}^{(1)} h_{p}^{(1) \mu \nu}+e^{\bar{\phi}_{D}^{(1)}}\left(h_{\mu \nu m}^{(2)}+\phi^{(2)} h_{\mu \nu m}^{(1)}\right)\left(h_{p}^{(2) \mu \nu}+\phi^{(2)} h_{p}^{(1) \mu \nu}\right)\right\} \\
& \left.-\frac{1}{12}(\bar{\Delta})^{1 / 2}\left\{e^{-\bar{\phi}_{D}^{(1)}} h_{\mu \nu \lambda}^{(1)} h^{(1) \mu \nu \lambda}+e^{\bar{\phi}_{D}^{(1)}}\left(h_{\mu \nu \lambda}^{(2)}+\phi^{(2)} h_{\mu \nu \lambda}^{(1)}\right)\left(h^{(2) \mu \nu \lambda}+\phi^{(2)} h^{(1) \mu \nu \lambda}\right)\right\}\right]
\end{array}
$$

where we have defined $\bar{\phi}_{D}^{(1)} \equiv \phi_{D}^{(1)}+\frac{1}{2} \log \Delta$. Also, $g_{m n}=e^{\frac{4}{D-2} \phi_{D}^{(1)}} \bar{g}_{m n}$ and so, $\Delta=$ $e^{2 \frac{(10-D)}{(D-2)} \phi_{D}^{(1)}} \bar{\Delta}$, with $\bar{\Delta}^{2} \equiv\left(\operatorname{det} \bar{g}_{m n}\right)$. Note that unlike $\hat{\phi}^{(1)}, \bar{\phi}_{D}^{(1)}$ is a D-dimensional field composed of D-dimensional scalars $\phi_{D}^{(1)}$ and $\Delta$. By defining

$$
\mathcal{M}_{D} \equiv\left(\begin{array}{cc}
\left(\phi^{(2)}\right)^{2} e^{\bar{\phi}_{D}^{(1)}}+e^{-\bar{\phi}_{D}^{(1)}} & \phi^{(2)} e^{\bar{\phi}_{D}^{(1)}} \\
\phi^{(2)} e^{\bar{\phi}_{D}^{(1)}} & e^{\bar{\phi}_{D}^{(1)}}
\end{array}\right)
$$

the action (18) can be expressed in a manifestly $\mathrm{SL}(2, \mathrm{R})$ invariant form

$$
\begin{aligned}
\int d^{D} x \sqrt{-\bar{g}_{D}}[ & \bar{R}_{D}+\frac{1}{4} \operatorname{tr} \partial_{\mu} \mathcal{M}_{D} \partial^{\mu} \mathcal{M}_{D}^{-1}+\frac{1}{8} \partial_{\mu} \log \bar{\Delta} \partial^{\mu} \log \bar{\Delta}+\frac{1}{4} \partial_{\mu} \bar{g}_{m n} \partial^{\mu} \bar{g}^{m n} \\
& -\frac{1}{4} \bar{g}_{m n} f_{\mu \nu}^{(3) m} f^{(3) \mu \nu, n}-\frac{1}{4}(\bar{\Delta})^{1 / 2} \bar{g}^{m p} \bar{g}^{n q} \partial_{\mu} \mathbf{b}_{m n}^{T} \mathcal{M}_{D} \partial^{\mu} \mathbf{b}_{p q} \\
& \left.-\frac{1}{4}(\bar{\Delta})^{1 / 2} \bar{g}^{m p} \mathbf{h}_{\mu \nu m}^{T} \mathcal{M}_{D} \mathbf{h}_{p}^{\mu \nu}-\frac{1}{12}(\bar{\Delta})^{1 / 2} \mathbf{h}_{\mu \nu \lambda}^{T} \mathcal{M}_{D} \mathbf{h}^{\mu \nu \lambda}\right]
\end{aligned}
$$

Here we have defined $\mathbf{b}_{m n} \equiv\left(\begin{array}{c}b_{m n}^{(1)} \\ b_{m n}^{(2)}\end{array}\right), \mathbf{h}_{\mu \nu m} \equiv\left(\begin{array}{c}h_{\mu \nu m}^{(1)} \\ h_{\mu \nu m}^{(2)}\end{array}\right)$, and $\mathbf{h}_{\mu \nu \lambda} \equiv\left(\begin{array}{c}h_{\mu \nu \lambda}^{(1)} \\ h_{\mu \nu \lambda}^{(2)}\end{array}\right)$. Now it is clear that the action (20) is invariant under the following global $\mathrm{SL}(2, \mathrm{R})$ transformation:

$$
\begin{aligned}
\mathcal{M}_{D} & \rightarrow \Lambda \mathcal{M}_{D} \Lambda^{T}, \quad \mathbf{b}_{m n} \rightarrow\left(\Lambda^{-1}\right)^{T} \mathbf{b}_{m n} \\
\left(\begin{array}{c}
a_{\mu m}^{(1)} \\
a_{\mu m}^{(2)}
\end{array}\right) & \equiv \mathbf{a}_{\mu m} \rightarrow\left(\Lambda^{-1}\right)^{T} \mathbf{a}_{\mu m}, \quad\left(\begin{array}{c}
b_{\mu \nu}^{(1)} \\
b_{\mu \nu}^{(2)}
\end{array}\right) \equiv \mathbf{b}_{\mu \nu} \rightarrow\left(\Lambda^{-1}\right)^{T} \mathbf{b}_{\mu \nu} \\
\bar{g}_{\mu \nu} & \rightarrow \bar{g}_{\mu \nu}, \quad \bar{g}_{m n} \rightarrow \bar{g}_{m n}, \quad \text { and } a_{\mu}^{(3) m} \rightarrow a_{\mu}^{(3) m}
\end{aligned}
$$

${ }^{\dagger}$ This corrects the inference drawn in ref.[18] about the $\mathrm{SL}(2, \mathrm{R})$ non-invariance of the $\mathrm{D}=9$ type IIB action. 
where $\Lambda=\left(\begin{array}{ll}a & b \\ c & d\end{array}\right)$, with $a d-b c=1$, is the $\operatorname{SL}(2, \mathrm{R})$ transformation matrix. The same transformation rules have been found in ref.[16] using different method. Note, however, that it is the complex scalar $\rho_{D}=\phi^{(2)}+i e^{-\bar{\phi}_{D}^{(1)}}$ which undergoes the usual fractional linear transformation under $(21)$, where $\rho_{D}$ involves $\bar{\phi}_{D}^{(1)}$ and not $\phi_{D}^{(1)}$, the D-dimensional dilaton. Also, note that it is $\bar{\Delta}$ which remains invariant under $\operatorname{SL}(2, \mathrm{R})$ transformation, whereas $\Delta$ changes. This follows from the fact that the internal metric $g_{m n}$ does not remain invariant under $\mathrm{SL}(2, \mathrm{R})$ transformation, whereas the scaled metric $\bar{g}_{m n}$ is invariant.

Let us then look at the special case of this $\mathrm{SL}(2, \mathrm{R})$ transformation i.e. the $\mathrm{Z}_{2}$ subgroup generated by $\Lambda=\left(\begin{array}{cc}0 & 1 \\ -1 & 0\end{array}\right)$. We have mentioned before for the ten dimensional case that it generates the strong-weak coupling duality when $\hat{\phi}^{(2)}=0$. For the D-dimensional action if we set $\phi^{(2)}=0$, the same $\Lambda$ generates the symmetry $\bar{\phi}_{D}^{(1)} \rightarrow-\bar{\phi}_{D}^{(1)}$. Since $\bar{\phi}_{D}^{(1)}$ is not the D-dimensional dilaton, this symmetry does not necessarily mean a strong-weak coupling duality symmetry in the D-dimensional theory. By demanding that

$$
\bar{\Delta}=e^{-2 \frac{(10-D)}{(D-2)} \phi_{D}^{(1)}} \Delta
$$

remains invariant under $\mathrm{Z}_{2}$ transformation, we find the transformation rules for the $\mathrm{D}$ dimensional dilaton $\phi_{D}^{(1)}$ and $\log \Delta$ as follows:

$$
\begin{aligned}
\phi_{D}^{(1)} & \rightarrow \frac{6-D}{4} \phi_{D}^{(1)}+\frac{2-D}{8} \log \Delta \\
\log \Delta & \rightarrow \frac{D-10}{2} \phi_{D}^{(1)}+\frac{D-6}{4} \log \Delta
\end{aligned}
$$

In particular, for $\mathrm{D}=10$, we get from $(23) \phi_{10}^{(1)} \rightarrow-\phi_{10}^{(1)}$ as expected. Also we note here that for $\mathrm{D} \leq 6, \phi_{D}^{(1)}$ does not change sign and so one might be tempted to think that in this case $\mathrm{Z}_{2}$ transformation does not produce the strong-weak coupling duality in the reduced theory. We point out that this inference is not quite correct as $\log \Delta$ also changes under $\mathrm{Z}_{2}$. If we express $\bar{\phi}_{D}^{(1)}$ in terms of $\operatorname{SL}(2, \mathrm{R})$ invariant quantity $\bar{\Delta}$, we find,

$$
\bar{\phi}_{D}^{(1)}=\frac{8}{D-2} \phi_{D}^{(1)}+\frac{1}{2} \log \bar{\Delta}
$$

Now demanding $\bar{\phi}_{D}^{(1)} \rightarrow-\bar{\phi}_{D}^{(1)}$ under $\mathrm{Z}_{2}$, we find the transformation rule for the Ddimensional dilaton as

$$
\phi_{D}^{(1)} \rightarrow-\phi_{D}^{(1)}+\frac{2-D}{8} \log \bar{\Delta}
$$

implying a strong-weak coupling duality in the D-dimensional theory apart from a constant scaling factor $(\bar{\Delta})^{(2-D) / 8}$. We have thus clarified how the $\mathrm{Z}_{2}$ subgroup of $\mathrm{SL}(2, \mathrm{R})$ 
group in the ten-dimensional theory induces a strong-weak coupling duality in the reduced theory.

Next we show that the $\mathrm{SL}(2, \mathrm{R})$ symmetry of the type IIB string theory is not only the symmetry of the truncated action (1), but also is a symmetry of the full theory compactified on torus. As the full type IIB action is not known we take the complete type IIA string effective action [18] and then compactify on a circle. We then make a T-duality transformation $[24,18]$ on this $9 \mathrm{D}$ action to convert it into type IIB action. This T-dual action will be shown to have the $\mathrm{SL}(2, \mathrm{R})$ invariance. The massless spectrum of the bosonic sector of type IIA string theory consists of a metric $\hat{g}_{\hat{\mu} \hat{\nu}}$, an antisymmetric tensor field $\hat{B}_{\hat{\mu} \hat{\nu}}^{(1)}$ and a dilaton $\hat{\phi}$ in the NS-NS sector and in the R-R sector it has a vector gauge field $\hat{A}_{\hat{\mu}}^{(1)}$ and a three-form antisymmetric tensor field $\hat{C}_{\hat{\mu} \hat{\nu} \hat{\lambda}}$ [21]. The full action in $\mathrm{D}=10$ is given in the following [18]:

$$
\begin{aligned}
& S_{\text {IIA }}^{(10)}=\int d^{10} \hat{x} \sqrt{-\hat{g}}\left[e^{-2 \hat{\phi}}\left(\hat{R}+4 \partial_{\hat{\mu}} \hat{\phi} \partial^{\hat{\mu}} \hat{\phi}-\frac{1}{12} \hat{H}_{\hat{\mu} \hat{\nu} \hat{\lambda}}^{(1)} \hat{H}^{(1) \hat{\mu} \hat{\nu} \hat{\lambda}}\right)-\frac{1}{4} \hat{F}_{\hat{\mu} \hat{\nu}}^{(1)} \hat{F}^{(1) \hat{\mu} \hat{\nu}}\right. \\
& -\frac{1}{12} \hat{F}_{\hat{\mu} \hat{\nu} \hat{\lambda} \hat{\rho}} \hat{F}^{\hat{\mu} \hat{\nu} \hat{\lambda} \hat{\rho}}+\frac{4}{(12)^{4}} \frac{\epsilon^{\hat{\mu}_{1} \ldots \hat{\mu}_{10}}}{\sqrt{-\hat{g}}}\left(3 \hat{F}_{\hat{\mu}_{1} \ldots \hat{\mu}_{4}} \hat{F}_{\hat{\mu}_{5} \ldots \hat{\mu}_{8}} \hat{B}_{\hat{\mu}_{9} \hat{\mu}_{10}}^{(1)}\right. \\
& \left.\left.-8 \hat{F}_{\hat{\mu}_{1} \ldots \hat{\mu}_{4}} \hat{H}_{\hat{\mu}_{5} \hat{\mu}_{6} \hat{\mu}_{7}} \hat{C}_{\hat{\mu}_{8} \hat{\mu}_{9} \hat{\mu}_{10}}\right)\right]
\end{aligned}
$$

where $\hat{H}_{\hat{\mu} \hat{\nu} \hat{\lambda}}^{(1)}=\left(\partial_{\hat{\mu}} \hat{B}_{\hat{\nu} \hat{\lambda}}^{(1)}+\right.$ cyc. in $\left.\hat{\mu} \hat{\nu} \hat{\lambda}\right), \hat{F}_{\hat{\mu} \hat{\nu}}^{(1)}=\partial_{\hat{\mu}} \hat{A}_{\hat{\nu}}^{(1)}-\partial_{\hat{\nu}} \hat{A}_{\hat{\mu}}^{(1)}$ and $\hat{F}_{\hat{\mu} \hat{\nu} \hat{\rho} \hat{\rho}}=\partial_{\hat{\mu}} \hat{C}_{\hat{\nu} \hat{\lambda} \hat{\rho}}-$ $\partial_{\hat{\nu}} \hat{C}_{\hat{\mu} \hat{\lambda} \hat{\rho}}+\partial_{\hat{\lambda}} \hat{C}_{\hat{\rho} \hat{\mu} \hat{\nu}}-\partial_{\hat{\rho}} \hat{C}_{\hat{\lambda} \hat{\mu} \hat{\nu}}+\left(\hat{F}_{\hat{\mu} \hat{\nu}}^{(1)} \hat{B}_{\hat{\lambda} \hat{\rho}}^{(1)}+\hat{F}_{\hat{\nu} \hat{\lambda}}^{(1)} \hat{B}_{\hat{\mu} \hat{\rho}}^{(1)}+\right.$ cyc. in $\left.\hat{\nu} \hat{\lambda} \hat{\rho}\right)$. Note that the first three terms in (26) i.e. the NS-NS terms couple to the dilaton, whereas, the fourth and the fifth terms are R-R terms and do not couple to the dilaton. But all these terms are dependent on the metric $\hat{g}_{\hat{\mu} \hat{\nu}}$. On the other hand, the last two terms are mixed terms and do not depend on the metric. In this sense these terms are topological. The reduced forms of these terms depend on the dimensionality of the theory. The reduction of this action (26) on a circle has already been performed in ref.[18]. We here write the $\mathrm{D}=9$ type IIA string effective action without the topological terms (the $\mathrm{SL}(2, \mathrm{R})$ invariance of the T-dual form of the topological terms will be considered later),

$$
\begin{aligned}
S_{\mathrm{IIA}}^{(9)}= & \int d^{9} x \sqrt{-g}\left[e ^ { - 2 \phi } \left(R+4 \partial_{\mu} \phi \partial^{\mu} \phi-\frac{1}{4} \partial_{\mu} \log \chi \partial^{\mu} \log \chi-\frac{1}{4} \chi F_{\mu \nu}^{(2)} F^{(2) \mu \nu}\right.\right. \\
& \left.-\frac{1}{4} \chi^{-1} F_{\mu \nu}^{(3)} F^{(3) \mu \nu}-\frac{1}{12} H_{\mu \nu \lambda}^{(1)} H^{(1) \mu \nu \lambda}\right)-\frac{1}{2} \chi^{-\frac{1}{2}} \partial_{\mu} \psi \partial^{\mu} \psi-\frac{1}{12} \chi^{\frac{1}{2}} F_{\mu \nu \lambda \rho} F^{\mu \nu \lambda \rho} \\
& -\frac{1}{12} \chi^{-\frac{1}{2}}\left(H_{\mu \nu \lambda}^{(2)}-\psi H_{\mu \nu \lambda}^{(1)}\right)\left(H^{(2) \mu \nu \lambda}-\psi H^{(1) \mu \nu \lambda}\right) \\
& \left.-\frac{1}{4} \chi^{\frac{1}{2}}\left(F_{\mu \nu}^{(1)}+\psi F_{\mu \nu}^{(2)}\right)\left(F^{(1) \mu \nu}+\psi F^{(2) \mu \nu}\right)\right]
\end{aligned}
$$


where the definitions of various reduced fields and their field-strengths are listed below:

$$
\begin{aligned}
& \hat{g}_{\hat{\mu} \hat{\nu}} \longrightarrow\left\{\begin{array}{l}
\hat{g}_{99}=g_{99}=\chi \\
\hat{g}_{\mu 9}=g_{\mu 9}=\chi A_{\mu}^{(2)} \\
\hat{g}_{\mu \nu}=g_{\mu \nu}+\chi A_{\mu}^{(2)} A_{\nu}^{(2)}
\end{array}\right. \\
& \hat{\phi}=\phi+\frac{1}{4} \log \chi \\
& \hat{A}_{\hat{\mu}}^{(1)} \longrightarrow\left\{\begin{array}{l}
A_{9}=\hat{A}_{9}=\psi \\
A_{\mu}^{(1)}=\hat{A}_{\mu}^{(1)}-\psi A_{\mu}^{(2)}
\end{array}\right. \\
& \hat{B}_{\hat{\mu} \hat{\nu}}^{(1)} \longrightarrow\left\{\begin{array}{l}
B_{\mu 9}^{(1)}=\hat{B}_{\mu 9}^{(1)}=A_{\mu}^{(3)} \\
B_{\mu \nu}^{(1)}=\hat{B}_{\mu \nu}^{(1)}+A_{\mu}^{(2)} A_{\nu}^{(3)}-A_{\nu}^{(2)} A_{\mu}^{(3)}
\end{array}\right. \\
& \hat{C}_{\hat{\mu} \hat{\nu} \hat{\lambda}} \longrightarrow\left\{\begin{array}{l}
C_{\mu \nu 9}=\hat{C}_{\mu \nu 9}=B_{\mu \nu}^{(2)}-\psi B_{\mu \nu}^{(1)}-\left(A_{\mu}^{(1)} A_{\nu}^{(3)}-A_{\nu}^{(1)} A_{\mu}^{(3)}\right) \\
C_{\mu \nu \lambda}=\hat{C}_{\mu \nu \lambda}-\left(A_{\mu}^{(2)} \hat{C}_{\nu \lambda 9}+\text { cyc. in } \mu \nu \lambda\right)
\end{array}\right.
\end{aligned}
$$

and the field strengths are:

$$
\begin{aligned}
F_{\mu \nu}^{(2)} & =\partial_{\mu} A_{\nu}^{(2)}-\partial_{\nu} A_{\mu}^{(2)}, \quad F_{\mu \nu}^{(3)}=\partial_{\mu} A_{\nu}^{(3)}-\partial_{\nu} A_{\mu}^{(3)}, \quad F_{\mu 9}=\partial_{\mu} \psi \\
F_{\mu \nu} & =F_{\mu \nu}^{(1)}+\psi F_{\mu \nu}^{(2)}, \quad H_{\mu \nu 9}^{(1)}=F_{\mu \nu}^{(3)} \\
H_{\mu \nu \lambda}^{(1)} & =\partial_{\mu} B_{\nu \lambda}^{(1)}-F_{\mu \nu}^{(2)} A_{\lambda}^{(3)}+\text { cyc. in } \mu \nu \lambda \\
& =\partial_{\mu} \bar{B}_{\nu \lambda}^{(1)}-\frac{1}{2}\left(F_{\mu \nu}^{(2)} A_{\lambda}^{(3)}+F_{\mu \nu}^{(3)} A_{\lambda}^{(2)}\right)+\text { cyc. in } \mu \nu \lambda
\end{aligned}
$$

we have defined

$$
\bar{B}_{\mu \nu}^{(1)} \equiv B_{\mu \nu}^{(1)}-\frac{1}{2}\left(A_{\mu}^{(2)} A_{\nu}^{(3)}-A_{\nu}^{(2)} A_{\mu}^{(3)}\right)
$$

Continuing with other field strengths,

$$
F_{\mu \nu \lambda 9}=\hat{F}_{\mu \nu \lambda 9}=H_{\mu \nu \lambda}^{(2)}-\psi H_{\mu \nu \lambda}^{(1)}
$$

where $H_{\mu \nu \lambda}^{(2)}$ is defined as,

$$
\begin{aligned}
H_{\mu \nu \lambda}^{(2)} & =\partial_{\mu} B_{\nu \lambda}^{(2)}+F_{\mu \nu}^{(3)} A_{\lambda}^{(1)}+\text { cyc. in } \mu \nu \lambda \\
& =\partial_{\mu} \bar{B}_{\nu \lambda}^{(2)}+\frac{1}{2}\left(F_{\mu \nu}^{(1)} A_{\lambda}^{(3)}+F_{\mu \nu}^{(3)} A_{\lambda}^{(1)}\right)+\text { cyc. in } \mu \nu \lambda
\end{aligned}
$$

where

$$
\bar{B}_{\mu \nu}^{(2)} \equiv B_{\mu \nu}^{(2)}-\frac{1}{2}\left(A_{\mu}^{(1)} A_{\nu}^{(3)}-A_{\nu}^{(1)} A_{\mu}^{(3)}\right)
$$

and finally,

$$
\begin{aligned}
F_{\mu \nu \lambda \rho}= & \partial_{\mu} \bar{C}_{\nu \lambda \rho}-\partial_{\nu} \bar{C}_{\mu \lambda \rho}+\partial_{\lambda} \bar{C}_{\rho \mu \nu}-\partial_{\rho} \bar{C}_{\lambda \mu \nu}+\left[F_{\mu \nu}^{(i)} \bar{B}_{\lambda \rho}^{(i)}+F_{\nu \lambda}^{(i)} \bar{B}_{\mu \rho}^{(i)}\right. \\
& \left.-\frac{1}{2} \epsilon^{i j} F_{\mu \nu}^{(3)} A_{\lambda}^{(i)} A_{\rho}^{(j)}-\frac{1}{2} \epsilon^{i j} F_{\nu \lambda}^{(3)} A_{\mu}^{(i)} A_{\rho}^{(j)}+\text { cyc. in } \nu \lambda \rho\right]
\end{aligned}
$$


where

$$
\bar{C}_{\mu \nu \lambda}=C_{\mu \nu \lambda}+\left[\frac{1}{2} \epsilon^{i j} A_{\mu}^{(i)} A_{\nu}^{(j)} A_{\lambda}^{(3)}+\text { cyc. in } \mu \nu \lambda\right]
$$

Here $i, j=1,2$ and $\epsilon^{12}=-\epsilon^{21}=1$. Note here that we have introduced $\bar{B}_{\mu \nu}^{(i)}$ fields. When we take T-duality transformation on the action (27), we will use the fact that $\bar{B}_{\mu \nu}^{(1)}$ does not transform whereas $\bar{B}_{\mu \nu}^{(2)}$ transforms in a non-local way. The T-duality transformation for which the NS-NS sector of the action (27) remains invariant is given by [18]:

$$
\tilde{\chi}=\chi^{-1}, \quad \tilde{A}_{\mu}^{(2)}=-A_{\mu}^{(3)}, \quad \tilde{A}_{\mu}^{(3)}=-A_{\mu}^{(2)}, \quad \tilde{g}_{\mu \nu}=g_{\mu \nu}, \quad \tilde{\phi}=\phi
$$

We will assume that both $H_{\mu \nu \lambda}^{(1)}$ and $H_{\mu \nu \lambda}^{(2)}$ do not transform under T-duality transformation. For $H^{(1)}$, it is clear from (34) and (41) that it is indeed invariant if $\bar{B}_{\mu \nu}^{(1)}$ is invariant, whereas, for $H^{(2)}$, we note from (37) that it will remain invariant if $\bar{B}_{\mu \nu}^{(2)}$ transforms in an appropriate non-local way. We will also assume that $F_{\mu \nu \lambda \rho}$ remains invariant under Tduality transformation, but it means from (39) that $\bar{C}_{\mu \nu \lambda}$ should transform appropriately. We should emphasize that these assumptions are made just for simplification. Now with these T-duality rules the action (27) changes as:

$$
\begin{aligned}
\tilde{S}_{\mathrm{IIA}}^{(9)^{\prime}}= & S_{\mathrm{IIB}}^{(9)^{\prime}} \\
= & \int d^{9} x \sqrt{-g}\left[e ^ { - 2 \phi } \left(R+4 \partial_{\mu} \phi \partial^{\mu} \phi-\frac{1}{4} \partial_{\mu} \log \chi \partial^{\mu} \log \chi-\frac{1}{4} \chi F_{\mu \nu}^{(2)} F^{(2) \mu \nu}\right.\right. \\
& \left.-\frac{1}{4} \chi^{-1} F_{\mu \nu}^{(3)} F^{(3) \mu \nu}-\frac{1}{12} H_{\mu \nu \lambda}^{(1)} H^{(1) \mu \nu \lambda}\right)-\frac{1}{2} \chi^{\frac{1}{2}} \partial_{\mu} \psi \partial^{\mu} \psi-\frac{1}{12} \chi^{-\frac{1}{2}} F_{\mu \nu \lambda \rho} F^{\mu \nu \lambda \rho} \\
& -\frac{1}{12} \chi^{\frac{1}{2}}\left(H_{\mu \nu \lambda}^{(2)}-\psi H_{\mu \nu \lambda}^{(1)}\right)\left(H^{(2) \mu \nu \lambda}-\psi H^{(1) \mu \nu \lambda}\right) \\
& \left.\quad-\frac{1}{4} \chi^{-\frac{1}{2}}\left(F_{\mu \nu}^{(1)}-\psi F_{\mu \nu}^{(3)}\right)\left(F^{(1) \mu \nu}-\psi F^{(3) \mu \nu}\right)\right]
\end{aligned}
$$

By rescaling the metric $g_{\mu \nu}=e^{\frac{4}{7} \phi} \bar{g}_{\mu \nu}$ and $\chi=e^{\frac{4}{7} \phi} \bar{\chi}$, we convert this part of the action (42) in the Einstein frame as follows:

$$
\begin{aligned}
\int d^{9} x \sqrt{-\bar{g}}\left(\bar{R}-\frac{1}{2} \partial_{\mu} \bar{\phi} \partial^{\mu} \bar{\phi}-\frac{1}{2} e^{2 \bar{\phi}} \partial_{\mu} \psi \partial^{\mu} \psi-\frac{7}{32} \partial_{\mu} \log \bar{\chi} \partial^{\mu} \log \bar{\chi}\right. \\
-\frac{1}{4} \bar{\chi} F_{\mu \nu}^{(2)} F^{(2) \mu \nu}-\frac{1}{12} \bar{\chi}^{-1 / 2} F_{\mu \nu \lambda \rho} F^{\mu \nu \lambda \rho} \\
-\frac{1}{4} \bar{\chi}^{-3 / 4}\left\{e^{-\bar{\phi}} F_{\mu \nu}^{(3)} F^{(3) \mu \nu}+e^{\bar{\phi}}\left(F_{\mu \nu}^{(1)}-\psi F_{\mu \nu}^{(3)}\right)\left(F^{(1) \mu \nu}-\psi F^{(3) \mu \nu}\right)\right\} \\
\left.-\frac{1}{12} \bar{\chi}^{1 / 4}\left\{e^{-\bar{\phi}} H_{\mu \nu \lambda}^{(1)} H^{(1) \mu \nu \lambda}+e^{\bar{\phi}}\left(H_{\mu \nu \lambda}^{(2)}-\psi H_{\mu \nu \lambda}^{(1)}\right)\left(H^{(2) \mu \nu \lambda}-\psi H^{(1) \mu \nu \lambda}\right)\right\}\right)
\end{aligned}
$$

Now by defining

$$
\mathcal{N}_{D} \equiv\left(\begin{array}{cc}
\psi^{2} e^{\bar{\phi}}+e^{-\bar{\phi}} & -\psi e^{\bar{\phi}} \\
-\psi e^{\bar{\phi}} & e^{\bar{\phi}}
\end{array}\right)
$$


the part of the action (43) can be written in a manifestly $\mathrm{SL}(2, \mathrm{R})$ invariant form as given below:

$$
\begin{array}{r}
\int d^{9} x \sqrt{-\bar{g}}\left(\bar{R}+\frac{1}{4} \operatorname{tr} \partial_{\mu} \mathcal{N}_{D} \partial^{\mu} \mathcal{N}_{D}^{-1}-\frac{7}{32} \partial_{\mu} \log \bar{\chi} \partial^{\mu} \log \bar{\chi}-\frac{1}{4} \bar{\chi} F_{\mu \nu}^{(2)} F^{(2) \mu \nu}\right. \\
\left.-\frac{1}{4} \bar{\chi}^{-3 / 4} \mathcal{F}_{\mu \nu}^{T} \mathcal{N}_{D} \mathcal{F}^{\mu \nu}-\frac{1}{12} \bar{\chi}^{1 / 4} \mathcal{H}_{\mu \nu \lambda}^{T} \mathcal{N}_{D} \mathcal{H}^{\mu \nu \lambda}-\frac{1}{12} \bar{\chi}^{-1 / 2} F_{\mu \nu \lambda \rho} F^{\mu \nu \lambda \rho}\right)
\end{array}
$$

where we have defined $\mathcal{F}_{\mu \nu} \equiv\left(\begin{array}{c}F_{\mu \nu}^{(3)} \\ F_{\mu \nu}^{(1)}\end{array}\right)$ and $\mathcal{H}_{\mu \nu \lambda} \equiv\left(\begin{array}{c}H_{\mu \nu \lambda}^{(1)} \\ H_{\mu \nu \lambda}^{(2)}\end{array}\right)$. So, the part of the action without the topological term is indeed invariant under the following global SL(2, R) transformation:

$$
\begin{aligned}
\mathcal{N}_{D} & \rightarrow \Lambda \mathcal{N}_{D} \Lambda^{T}, \quad \bar{g}_{\mu \nu} \rightarrow \bar{g}_{\mu \nu}, \quad \bar{\chi} \rightarrow \bar{\chi}, \quad A_{\mu}^{(2)} \rightarrow A_{\mu}^{(2)} \\
\left(\begin{array}{c}
A_{\mu}^{(3)} \\
A_{\mu}^{(1)}
\end{array}\right) & \equiv \mathcal{A}_{\mu} \rightarrow\left(\Lambda^{-1}\right)^{T} \mathcal{A}_{\mu}, \quad \mathcal{H}_{\mu \nu \lambda} \rightarrow\left(\Lambda^{-1}\right)^{T} \mathcal{H}_{\mu \nu \lambda} \\
F_{\mu \nu \lambda \rho} & \rightarrow F_{\mu \nu \lambda \rho}
\end{aligned}
$$

Note also that here $A_{\mu}^{(1)}, A_{\mu}^{(2)}, A_{\mu}^{(3)}$ play the same role as $a_{\mu}^{(2)}, a_{\mu}^{(3)}$ and $a_{\mu}^{(1)}$ respectively in the truncated version of type IIB theory as discussed earlier and so, those fields can be identified. Similarly, $H_{\mu \nu \lambda}^{(1)}, H_{\mu \nu \lambda}^{(2)}$ fields play the same role as $h_{\mu \nu \lambda}^{(1)}, h_{\mu \nu \lambda}^{(2)}$ in type IIB theory.

We now look at the topological part of the action (26) and show that this part is also $\mathrm{SL}(2, \mathrm{R})$ invariant. The dimensional reduction of the topological part on a circle has also been obtained in ref.[18] and the explicit form is given below:

$$
\begin{aligned}
S_{\text {IIA }}^{(9) "}= & \int d^{9} x \frac{1}{2(12)^{3}} \epsilon^{\mu_{1} \ldots \mu_{9}}\left(F_{\mu_{1} \ldots \mu_{4}} F_{\mu_{5} \ldots \mu_{8}} A_{\mu_{9}}^{(3)}-4 F_{\mu_{1} \ldots \mu_{4}} \epsilon^{i j} H_{\mu_{5} \mu_{6} \mu_{7}}^{(i)} \bar{B}_{\mu_{8} \mu_{9}}^{(j)}\right. \\
& +4 F_{\mu_{1} \ldots \mu_{4}} H_{\mu_{5} \mu_{6} \mu_{7}}^{(i)} A_{\mu_{8}}^{(i)} A_{\mu_{9}}^{(3)}+2 \epsilon^{i j} H_{\mu_{1} \mu_{2} \mu_{3}}^{(i)} H_{\mu_{4} \mu_{5} \mu_{6}}^{(j)} \bar{C}_{\mu_{7} \mu_{8} \mu_{9}}+4 F_{\mu_{1} \ldots \mu_{4}} F_{\mu_{5} \mu_{6}}^{(3)} \bar{C}_{\mu_{7} \mu_{8} \mu_{9}} \\
& \left.+6 \epsilon^{i j} H_{\mu_{1} \mu_{2} \mu_{3}}^{(i)} H_{\mu_{4} \mu_{5} \mu_{6}}^{(j)} A_{\mu_{7}}^{(k)} \bar{B}_{\mu_{8} \mu_{9}}^{(k)}+12 F_{\mu_{1} \ldots \mu_{4}} F_{\mu_{5} \mu_{6}}^{(3)} A_{\mu_{7}}^{(i)} \bar{B}_{\mu_{8} \mu_{9}}^{(i)}\right)
\end{aligned}
$$

We then make, as before, the T-duality transformation (41) and the rules given afterwards to convert the topological part of the type IIA action to type IIB action as follows:

$$
\begin{aligned}
& \tilde{S}_{\text {IIA }}^{(9)^{\prime \prime}}=S_{\mathrm{IIB}}^{(9)^{\prime \prime}} \\
= & \int d^{9} x \frac{1}{2(12)^{3}} \epsilon^{\mu_{1} \ldots \mu_{9}}\left[-F_{\mu_{1} \ldots \mu_{4}} F_{\mu_{5} \ldots \mu_{8}} A_{\mu_{9}}^{(2)}-F_{\mu_{1} \ldots \mu_{4}} H_{\mu_{5} \mu_{6} \mu_{7}}^{(1)} \tilde{\bar{B}}_{\mu_{8} \mu_{9}}^{(2)}\right. \\
+ & F_{\mu_{1} \ldots \mu_{4}} H_{\mu_{5} \mu_{6} \mu_{7}}^{(2)} \bar{B}_{\mu_{8} \mu_{9}}^{(1)}-4 F_{\mu_{1} \ldots \mu_{4}} H_{\mu_{5} \mu_{6} \mu_{7}}^{(1)} A_{\mu_{8}}^{(1)} A_{\mu_{9}}^{(2)}+4 F_{\mu_{1} \ldots \mu_{4}} H_{\mu_{5} \mu_{6} \mu_{7}}^{(2)} A_{\mu_{8}}^{(3)} A_{\mu_{9}}^{(2)} \\
+ & 2 H_{\mu_{1} \mu_{2} \mu_{3}}^{(1)} H_{\mu_{4} \mu_{5} \mu_{6}}^{(2)} \tilde{\bar{C}}_{\mu_{7} \mu_{8} \mu_{9}}-2 H_{\mu_{1} \mu_{2} \mu_{3}}^{(2)} H_{\mu_{4} \mu_{5} \mu_{6}}^{(1)} \tilde{\bar{C}}_{\mu_{7} \mu_{8} \mu_{9}}-4 F_{\mu_{1} \ldots \mu_{4}} F_{\mu_{5} \mu_{6}}^{(2)} \tilde{\bar{C}}_{\mu_{7} \mu_{8} \mu_{9}} \\
+ & 6\left(H_{\mu_{1} \mu_{2} \mu_{3}}^{(1)} H_{\mu_{4} \mu_{5} \mu_{6}}^{(2)} A_{\mu_{7}}^{(1)} \bar{B}_{\mu_{8} \mu_{9}}^{(1)}-H_{\mu_{1} \mu_{2} \mu_{3}}^{(1)} H_{\mu_{4} \mu_{5} \mu_{6}}^{(2)} A_{\mu_{7}}^{(3)} \tilde{\bar{B}}_{\mu_{8} \mu_{9}}^{(2)}-H_{\mu_{1} \mu_{2} \mu_{3}}^{(2)} H_{\mu_{4} \mu_{5} \mu_{6}}^{(1)} A_{\mu_{7}}^{(1)} \bar{B}_{\mu_{8} \mu_{9}}^{(1)}\right.
\end{aligned}
$$




$$
\left.\left.+H_{\mu_{1} \mu_{2} \mu_{3}}^{(2)} H_{\mu_{4} \mu_{5} \mu_{6}}^{(1)} A_{\mu_{7}}^{(3)} \tilde{\bar{B}}_{\mu_{8} \mu_{9}}^{(2)}\right)-12\left(F_{\mu_{1} \ldots \mu_{4}} F_{\mu_{5} \mu_{6}}^{(2)} A_{\mu_{7}}^{(1)} \bar{B}_{\mu_{8} \mu_{9}}^{(1)}+F_{\mu_{1} \ldots \mu_{4}} F_{\mu_{5} \mu_{6}}^{(2)} A_{\mu_{7}}^{(3)} \tilde{\bar{B}}_{\mu_{8} \mu_{9}}^{(2)}\right)\right]
$$

Here, $\tilde{\bar{B}}_{\mu \nu}^{(2)}$ and $\tilde{\bar{C}}_{\mu \nu \lambda}$ denote the T-dual forms of the corresponding fields $\bar{B}_{\mu \nu}^{(2)}, \bar{C}_{\mu \nu \lambda}$ whose explicit forms are not important. If we now introduce $\mathrm{SL}(2, \mathrm{R})$ metric $\Sigma=\left(\begin{array}{cc}0 & i \\ -i & 0\end{array}\right)$ which satisfies $\Lambda \Sigma \Lambda^{T}=\Sigma$ and $\Sigma \Lambda \Sigma=\Lambda^{-1}$ for any $\operatorname{SL}(2, \mathrm{R})$ matrix $\Lambda$, then in terms of $\Sigma,(48)$ can be written in a manifestly $\mathrm{SL}(2, \mathrm{R})$ invariant form as given below:

$$
\begin{aligned}
& \int d^{9} x \frac{1}{2(12)^{3}} \epsilon^{\mu_{1} \ldots \mu_{9}}\left(-F_{\mu_{1} \ldots \mu_{4}} F_{\mu_{5} \ldots \mu_{8}} A_{\mu_{9}}^{(2)}-i F_{\mu_{1} \ldots \mu_{4}} \mathcal{H}_{\mu_{5} \mu_{6} \mu_{7}}^{T} \Sigma \mathcal{B}_{\mu_{8} \mu_{9}}\right. \\
& -4 i F_{\mu_{1} \ldots \mu_{4}} \mathcal{H}_{\mu_{5} \mu_{6} \mu_{7}}^{T} \Sigma \mathcal{A}_{\mu_{8}} A_{\mu_{9}}^{(2)}+2 i \mathcal{H}_{\mu_{1} \mu_{2} \mu_{3}}^{T} \Sigma \mathcal{H}_{\mu_{4} \mu_{5} \mu_{6}} \tilde{\bar{C}}_{\mu_{7} \mu_{8} \mu_{9}}-4 F_{\mu_{1} \ldots \mu_{4}} F_{\mu_{5} \mu_{6}}^{(2)} \tilde{\bar{C}}_{\mu_{7} \mu_{8} \mu_{9}} \\
& \left.+6 \mathcal{H}_{\mu_{1} \mu_{2} \mu_{3}}^{T} \Sigma \mathcal{H}_{\mu_{4} \mu_{5} \mu_{6}} \mathcal{A}_{\mu_{7}}^{T} \Sigma \mathcal{B}_{\mu_{8} \mu_{9}}+12 i F_{\mu_{1} \ldots \mu_{4}} F_{\mu_{5} \mu_{6}}^{(2)} \mathcal{A}_{\mu_{7}}^{T} \Sigma \mathcal{B}_{\mu_{8} \mu_{9}}\right)
\end{aligned}
$$

Thus it is clear that the T-dual form of the topological part of the action (26) compactified on a circle is $\mathrm{SL}(2, \mathrm{R})$ invariant under the transformations (46) alongwith

$$
\tilde{\bar{C}}_{\mu \nu \lambda} \rightarrow \tilde{\bar{C}}_{\mu \nu \lambda}
$$

Note that $\mathcal{H}_{\mu \nu \lambda}$ transformation in (46) implies that $\mathcal{B}_{\mu \nu} \equiv\left(\begin{array}{c}\bar{B}_{\mu \nu}^{(1)} \\ \tilde{\bar{B}}_{\mu \nu}^{(2)}\end{array}\right)$ transforms as $\mathcal{B}_{\mu \nu} \rightarrow\left(\Lambda^{-1}\right)^{T} \mathcal{B}_{\mu \nu}$. So, we have shown that the T-dual form of the full type IIA action in 9D i.e. the full type IIB string effective action in $9 \mathrm{D}$ is invariant under the $\mathrm{SL}(2, \mathrm{R})$ transformations (46) and (50).

To conclude, we have studied the toroidal compactification of a truncated version (when the self-dual five-form field strength is zero) of the type IIB string effective action in the string frame. As we finally converted the reduced action in the Einstein frame by conformal rescaling of the metric we have recovered the $\mathrm{SL}(2, \mathrm{R})$ invariance of the reduced action as a consequence of the same symmetry in ten dimensions. We have obtained the transformation properties of the various fields and compared with the recently obtained results of toroidal compactification of the same type IIB action in the Einstein frame. Since the $\mathrm{SL}(2, \mathrm{R})$ matrix $\mathcal{M}_{D}$ in eq.(19) involved in the process of showing the invariance does not contain the D-dimensional dilaton, the issue of strong-weak coupling duality symmetry under a $Z_{2}$ subgroup of this $\mathrm{SL}(2, \mathrm{R})$ group becomes confusing. We have clarified this point and have shown how the $\mathrm{Z}_{2}$ subgroup produces the strong-weak coupling duality in the reduced theory. We have also shown how $\mathrm{SL}(2, \mathrm{R})$ invariance can be understood in the full type IIB string theory. In the absence of the complete type IIB action, we have 
taken a T-dual version of the complete type IIA action compactified on a circle. In this simplest case, we were able to show that the full action is indeed $\mathrm{SL}(2, \mathrm{R})$ invariant. It is quite involved to study the general case because of the complication of the topological terms whose forms are dependent on the dimensionality of the reduced theory. But, the invariance in 9D suggests that the full type IIB string effective action is $\mathrm{SL}(2, \mathrm{R})$ invariant. Using this symmetry it will be interesting to find the $\mathrm{SL}(2, \mathrm{Z})$ multiplets of various classical p-brane solutions of lower dimensional type II string theory.

\section{References:}

1. C. Hull and P. Townsend, Nucl. Phys. B438 (1995) 109.

2. C. Hull, Phys. Lett. B357 (1995) 545.

3. J. H. Schwarz, Phys. Lett. B360 (1995) 13; E: Phys. Lett. B364 (1995) 252; Superstring Dualities, hep-th/9509148.

4. A. Font, L. Ibanez, D. Lust and F. Quevedo, Phys. Lett. B249 (1990) 35; S. J. Rey, Phys. Rev D43 (1991) 35.

5. A. Sen, Int. J. Mod. Phys. A9 (1994) 3707; J. H. Schwarz, String Theory Symmetries, hep-th/9503127.

6. J. H. Schwarz, Phys. Lett. B367 (1996) 97.

7. J. H. Schwarz, Lectures on Superstring and M-Theory Dualities, hep-th/9607201.

8. E. Witten, Nucl. Phys. B460 (1996) 335.

9. A. Dabholkar, Ten Dimensional Heterotic String as a Soliton, hep-th/9506160; A. Sen, Unification of String Dualities, hep-th/9609176.

10. P. Aspinwall, Some Relationships Between Dualities in String Theory, in Proc. Trieste Conf. on "S-Duality and Mirror Symmetry", hep-th/9508154.

11. C. Vafa, Nucl. Phys. B469 (1996) 403.

12. J. Scherk and J. Schwarz, Nucl. Phys. B153 (1979) 61. 
13. E. Cremmer, in Supergravity, 1981 eds. S. Ferrara and J. G. Taylor (Cambridge University Press, 1982).

14. J. H. Schwarz, Nucl. Phys. B226 (1983) 269.

15. P. Howe and P. West, Nucl. Phys. B238 (1984) 181.

16. J. Maharana, S-Duality and Compactification of Type IIB Superstring Action, hepth/9703009.

17. E. Bergshoeff, C. M. Hull and T. Ortin, Nucl. Phys. B451 (1995) 547.

18. A. Das and S. Roy, Nucl. Phys. B482 (1996) 119.

19. M Dine, P. Huet and N. Seiberg, Nucl. Phys. B322 (1989) 301.

20. J. Dai, R. G. Leigh and J. Polchinski, Mod. Phys. Lett. A4 (1989) 2073.

21. M. B. Green, J. Schwarz and E. Witten, Superstring Theory, Vols. I and II, Cambridge University Press, 1987.

22. E. Cremmer and B. Julia, Nucl. Phys. B159 (1979) 141.

23. J. Maharana and J. Schwarz, Nucl. Phys. B390 (1993) 3; S. F. Hassan and A. Sen, Nucl. Phys. B375 (1992) 103.

24. T. Buscher, Phys. Lett. B194 (1987) 59; Phys. Lett. B201 (1988) 466. 